\title{
Diffusion Resonances in Action Space for an Atom Optics Kicked Rotor with Decoherence
}

\author{
A.J. Daley, A.S. Parkins, R. Leonhardt, and S.M. Tan \\ Department of Physics, University of Auckland, Private Bag 92019, Auckland, New Zealand
}

(Dated: 1 August 2001)

\begin{abstract}
We numerically investigate momentum diffusion rates for the pulse kicked rotor across the quantum to classical transition as the dynamics are made more macroscopic by increasing the total system action. For initial and late time rates we observe an enhanced diffusion peak which shifts and scales with changing kick strength, and we also observe distinctive peaks around quantum resonances. Our investigations take place in the context of a system of ultracold atoms which is coupled to its environment via spontaneous emission decoherence, and the effects should be realisable in ongoing experiments.
\end{abstract}

PACS numbers: $05.45 . \mathrm{Mt}, 03.65 . \mathrm{Yz}, 42.50 . \mathrm{Lc}$

The transition from quantum to classical behaviour in nonlinear dynamical systems has been a topic of much interest in recent years, motivated by the large differences that exist between the behaviour of such systems in the two regimes. The $\delta$-Kicked Rotor(DKR) is a prime example - in particular, coherence effects in the quantum DKR completely suppress classical diffusion 近, 2. The quantum DKR is also very interesting because it has been beautifully demonstrated in experiments which probe the momentum distribution of a cloud of laser-cooled atoms interacting with a pulsed standing wave of near resonant light [3]. These experiments necessarily involve a generalisation of the DKR to kicks of finite length, and it is this system, the Kicked Rotor(KR) which we investigate in this paper.

There have been numerous studies, both theoretical (see for example [4, 5, 6, 7, 8]) and experimental [9, 10, 11, 12, 13], of the role of decoherence in the quantum to classical transition for the Kicked Rotor. As with any real quantum system, the atom optics KR couples to its environment, resulting in a loss of phase coherence. In the case we consider here, this coupling is between the atoms and the vacuum electromagnetic field, and results in atomic spontaneous emissions and concomitant random momentum recoils.

Most work in the past has focussed on changing the level of this decoherence and observing the effects on momentum diffusion rates and distributions, looking in particular at how increased levels of decoherence "drive" the quantum system towards classical behaviour. In this paper we focus instead on what happens when we fix the level of decoherence in our real quantum system, and then make the dynamics more macroscopic by varying the total action in the system; that is, by varying the effective Planck's constant. In so doing we find dramatic structures in the momentum diffusion rates, similar to those found recently for the DKR with a continuous position measurement [14], only here we consider a specific experimental configuration.

The system we model is a cloud of ultracold Caesium atoms (of initial temperature $\approx 10 \mu K$ ) which interact with a standing wave of laser light of frequency $\omega_{l}$, detuned far from the frequency $w_{0}$ of the $6 S_{1 / 2} \rightarrow 6 P_{3 / 2}$ atomic transition. The laser is pulsed with period $T$ and pulse profile $f(t)$. If the detuning is large, the internal atomic dynamics can be eliminated, and the resulting single particle Hamiltonian (for just the external degrees of freedom) is [3]

$$
\hat{H}=\frac{\hat{p}^{2}}{2 m}-\frac{\hbar \Omega_{e f f}}{8} \cos \left(2 k_{l} \hat{x}\right) \sum_{n=0}^{N} f(t-n T),
$$

where $\hat{x}$ and $\hat{p}$ are the atomic position and momentum operators, respectively, and $k_{l}$ is the wave number of the laser light. The effective potential strength, $\Omega_{e f f}=\Omega^{2}\left(s_{45} / \delta_{45}+s_{44} / \delta_{44}+s_{43} / \delta_{43}\right)$, accounts for the different dipole transitions between hyperfine levels in the Caesium atoms $\left(\mathrm{F}=4 \rightarrow \mathrm{F}^{\prime}=5,4,3\right)$, with $\delta_{i j}$ the corresponding detunings, and $\Omega / 2$ the (single-beam) resonant Rabi frequency. If we assume equal populations in all Zeeman sublevels, then $s_{45}=\frac{11}{27}, s_{44}=\frac{7}{36}$, and $s_{43}=\frac{7}{108}$. We can rewrite this Hamiltonian in appropriate dimensionless units as

$$
\hat{H}^{\prime}=\frac{\hat{\rho}^{2}}{2}-k \cos \hat{\phi} \sum_{n=0}^{\infty} f\left(t^{\prime}-n\right),
$$

which is the Hamiltonian for the standard kicked rotor system. Here, $\hat{\phi}=2 k_{l} \hat{x}, \hat{\rho}=2 k_{l} T \hat{p} / m, t^{\prime}=t / T$, and $\hat{H}^{\prime}=\left(4 k_{l}^{2} T^{2} / m\right) \hat{H}$. The classical stochasticity parameter is given by $\kappa=\Omega_{e f f} \omega_{R} T \tau_{p}$, where $\tau_{p}$ is the pulse length and $\omega_{R}=\hbar k_{l}^{2} / 2 m$. In our work $f\left(t^{\prime}\right)$ is generally a square pulse, i.e., $f\left(t^{\prime}\right)=1$ for $0<t^{\prime}<\alpha$, where $\alpha=\tau_{p} / T$. Note that $k=\kappa / \alpha$. In these units, we have $[\hat{\phi}, \hat{\rho}]=i k$, with $k=8 \omega_{R} T$. Thus the quantum nature of the system is reflected by an effective Planck's constant, $k$, which scales as we change the total action in the system by altering the pulse period $T$.

Decoherence occurs in the form of spontaneous emission events, which occur when the atoms absorb light 
from the standing wave [9]. It is assumed that momentum distributions in orthogonal directions remain independent, and thus the system remains effectively one dimensional. We characterise the level of this decoherence by the probability of spontaneous emission per kick, $\eta$. Given the large detuning, i.e., $\Omega_{e f f} / \delta \ll 1$, this process may be modelled by the master equation for the density operator $\hat{w}$ of the system [8]

$$
\begin{aligned}
\dot{\hat{w}}= & -i[\hat{H}, \hat{w}]-\frac{\eta}{\alpha} \sum_{n=0}^{N} f(t-n)\left[\cos ^{2}(\hat{\phi} / 2), \hat{w}\right]_{+} \\
& +2 \frac{\eta}{\alpha} \sum_{n=1}^{N} f(t-n) \int_{-1}^{1} d u N(u) e^{i u \hat{\phi} / 2} \\
& \times \cos (\hat{\phi} / 2) \hat{w} \cos (\hat{\phi} / 2) e^{-i u \hat{\phi} / 2},
\end{aligned}
$$

where $N(u)$ is the distribution of recoil momenta projected onto the standing wave axis, and $[., .]_{+}$denotes an anti-commutator. We have also modelled spontaneous emission events in which the atoms absorb light from counterpropagating beams of oppositely circularly polarised light, which interact with the atoms continuously [10]. This leads to emission events which are independent of position, and which may occur at times other than during a kick. The results obtained for the two types of spontaneous emission noise are very similar, and so we only present the results for the first type here.
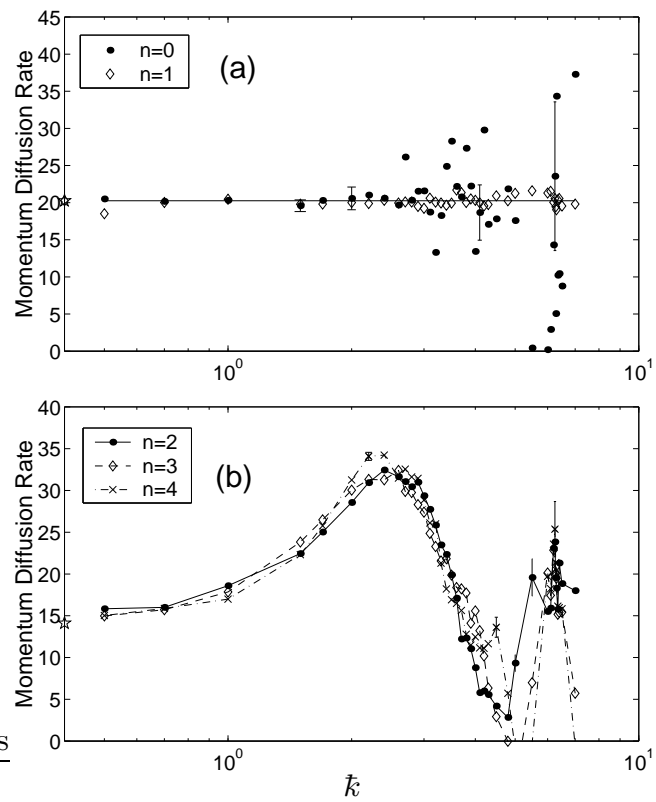

FIG. 1: Momentum diffusion rates $D(n)$ in the quantum kicked rotor as a function of the effective Planck's constant, $k$ for (a) the first two kicks $(n=0$ and $n=1)$ and (b) the third, fourth and fifth kicks $(n=2, n=3$ and $n=4)$, with $\eta=10 \%, \kappa=9$, and $\alpha=0.005$. Classical values are marked on the vertical axis.

We simulate Eq.(3) using the method of quantum trajectories, as described in Ref. [8]. We choose initial momentum eigenstates from a Gaussian distribution of width $\sigma_{\rho} / \hbar=\sigma_{p} / 2 \hbar k_{l}=4$ (which reflects the initial temperature distribution) and we take an incoherent average over the final momentum distributions. We choose $\alpha$ to be small so that the effects of KAM boundaries are not important [9]. Typically we use 1000 trajectories, and we calculate statistical errors (shown for some points in the figures) based on dividing these trajectories equally into 10 groups and computing errors in the means. We are primarily interested in the momentum diffusion rate, which is defined as the change in the kinetic energy from one kick to the next, $2 D(n)=<\hat{\rho}_{n+1}^{2}>-<\hat{\rho}_{n}^{2}>$, where we denote $\hat{\rho}_{0}=\hat{\rho}\left(t^{\prime}=0\right), \hat{\rho}_{1}=\hat{\rho}\left(t^{\prime}=1\right)$ etc.

Our simulation results for the momentum diffusion rates across the first five kicks are shown in Fig. 1. Aside from some noise in the simulations for larger values of $k$, we see that for the first two kicks the diffusion rates are essentially constant with respect to $k$. This is the quantum version of the quasilinear behaviour known to exist classically in the kicked rotor system. In fact one can show (for the DKR) that for a uniform initial distribution of positions, $2 D(0)=\kappa^{2} / 2$ (shown as a solid line in Fig. 1(a)). Similarly, if we also assume an initial Gaussian momentum distribution of standard deviation $\sigma_{\rho}$, it can be shown that

$$
\begin{aligned}
2 D(1)= & \frac{1}{2} \kappa^{2}\left(1-J_{2}\left(K_{2 q}\right) e^{-2 \sigma_{\rho}^{2}}\right)-2 \kappa J_{1}\left(K_{q}\right) \sigma_{\rho}^{2} e^{-\frac{\sigma_{\rho}^{2}}{2}} \\
& +\kappa^{2}\left(J_{0}\left(K_{q}\right)-J_{2}\left(K_{q}\right)\right) \cos (\kappa / 2) e^{-\frac{\sigma_{\rho}^{2}}{2}}
\end{aligned}
$$

where $J_{n}$ is an ordinary Bessel function of order $n, K_{q}=$ $2 \kappa \sin (k / 2) / \hbar$ and $K_{2 q}=2 \kappa \sin (\hbar) / \hbar$. For sufficiently large $\sigma_{\rho}$, this reduces to the same result as for the first kick, which can be seen for our system in Fig. 1](a).

After the second kick, the system settles down into its initial quantum diffusion period, where for a small time the system exhibits classical-like diffusion, with a relatively constant momentum diffusion rate, $D_{q}$. As can be seen from Fig. Il(b), the $k$ dependence of this rate is quite remarkable. We observe an enhanced diffusion peak (or resonance) around $k=3$ which shifts to the right and increases in magnitude as we increase $\kappa$ (See Fig. 2). There is also a peak in the diffusion rates near the quantum resonance at $k=2 \pi$ [15]. (This structure also occurs at larger multiples of $2 \pi$ ). The quantum diffusion rates that we observe in this regime agree well with those predicted by Shepelyansky under the conditions $k \geq 1$ and $\kappa \gg k$ [16], i.e.,

$$
D_{q}=\frac{\kappa^{2}}{2}\left(\frac{1}{2}-J_{2}\left(K_{q}\right)-J_{1}^{2}\left(K_{q}\right)+J_{2}^{2}\left(K_{q}\right)+J_{3}^{2}\left(K_{q}\right)\right) .
$$

This can be seen in Fig. 2, where we plot the average of the curves for $D(2-5)$. There is surprisingly good agreement for the quantum resonance peak and for the position of the enhanced diffusion peak, especially considering that the condition $\kappa \gg k$ does not hold for our large 
$\hbar$ values. The discrepancy in the height of the peak is created mainly by our choice to average over the diffusion rates from 4 different kicks. It is a qualitative decision as to when the system has really settled into the initial quantum diffusion regime, but averaging over $D(2-5)$ as we do gives us an objective estimate of the corresponding diffusion rate. However, in some cases (particularly near the maximum of the enhanced diffusion peak), the diffusion rate will already have begun to decrease towards the late time diffusion regime before the sixth kick.

The initial quantum diffusion period lasts for a small number of kicks, after which the diffusion rate begins to decrease. In the absence of noise, the system settles into a localised state [3], and $D(n) \rightarrow 0$ as $n \rightarrow \infty$. However, the onset of dynamical localisation is a coherence effect, and in the presence of decoherence the system settles into a late time diffusion regime where $D(n) \rightarrow D_{\infty} \neq 0$ as $n \rightarrow \infty$.

Our simulation results for these late time diffusion rates are shown for varying levels of decoherence, $\eta$, in Fig. 3, and for varying $\kappa$ in Fig. 4. Again we observe an enhanced diffusion peak (or resonance) which shifts and scales with increasing $\kappa$, as well as a much more narrow peak near the quantum resonance at $k=2 \pi$. The most notable feature here is that for appropriate values of $\kappa$ and $\eta$, the momentum diffusion rates near the top of the enhanced diffusion peak are actually larger than the corresponding classical values (marked on the graph), even if we account for added momentum diffusion due to spontaneous emission in the classical system.

The late time diffusion rates may be approximated by the formula [6, 9

$$
D_{\infty}=\sum_{n=0}^{\infty} \eta(1-\eta)^{n} D_{0}(n),
$$

where $D_{0}(n)$ is the diffusion rate at the $n$th kick for a KR without decoherence. Essentially, we assume here that dynamical correlations over particular time intervals are suppressed by a factor expressing the probability that a spontaneous emission occurs within that time interval. The correlations taken over a set number of kicks give rise to the diffusion rates seen in the $\mathrm{KR}$ without decoherence after that number of kicks. Hence, we take a weighted average over the diffusion rates as the KR settles down, where the weighting for $D_{0}(n)$ gives the probability that the first spontaneous emission event occurs during kick number $n+1$, i.e., $\eta(1-\eta)^{n}$. The early time diffusion rates are thus "locked in" by the loss of phase coherence.

In Fig. 目 the simulation results for $D_{\infty}$ are plotted as points, and calculations of the right hand side of Eq.(6) based on calculations of $D_{0}(n)$ from simulations of the KR without decoherence are shown as solid lines. There is very good agreement between the two sets of values, especially for the enhanced diffusion peak. Note that the values given by the solid lines contain statistical errors

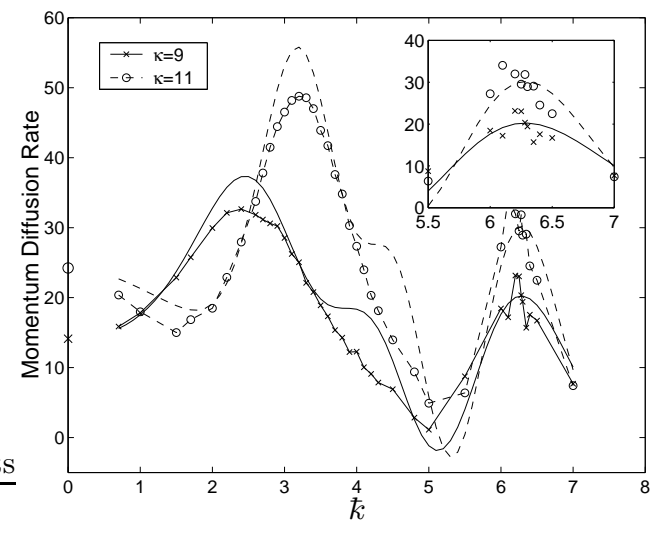

FIG. 2: Initial quantum diffusion rates in the kicked rotor for varying values of $\kappa$ and $k$, with $\eta=10 \%$ and $\alpha=0.005$. The points show simulation results (an average over $D(2-5)$ ), while the lines show Shepelyansky's formula, Eq.(周). Classical values are marked as points for $\hbar=0$. Note the use of a linear scale for $k$.

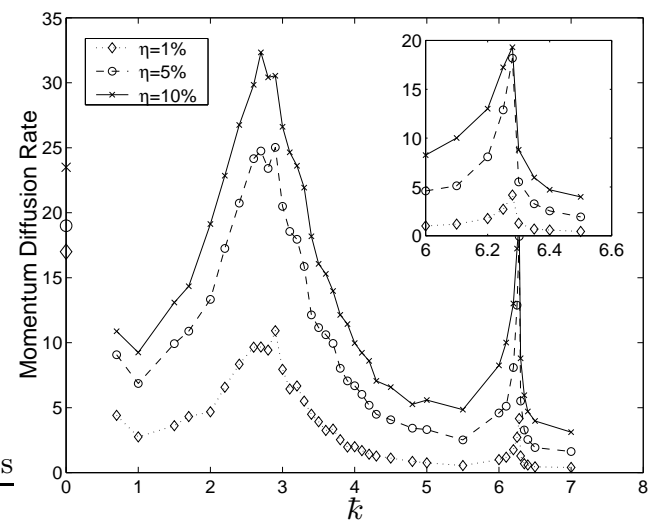

FIG. 3: Late time momentum diffusion rates in the quantum kicked rotor for varying values of $\eta$ and $k$, with $\kappa=10$ and $\alpha=0.005$. The insert shows the peak near the quantum resonance at $k=2 \pi$, and classical values are marked for $k=0$. Note the use of a linear scale for $k$.

from the simulations of $D_{0}(n)$ which are comparable in magnitude to those displayed in the figure. The level of agreement indicates that the model associated with Eq.(6) works very well for the late time diffusion rates.

Late time diffusion rates greater than the corresponding classical rates occur because quantum correlations cause the initial quantum diffusion rates to be higher than the corresponding classical rates in the appropriate cases, and these higher rates become locked in by the loss of phase coherence. These decoherence effects are thus much more important than the more direct increase in momentum diffusion due to the recoil in a spontaneous emission process which may be accounted for classically as well as quantum mechanically.

It is possible to find an analytical expression for $D_{\infty}$ which agrees well with the simulation results over a 


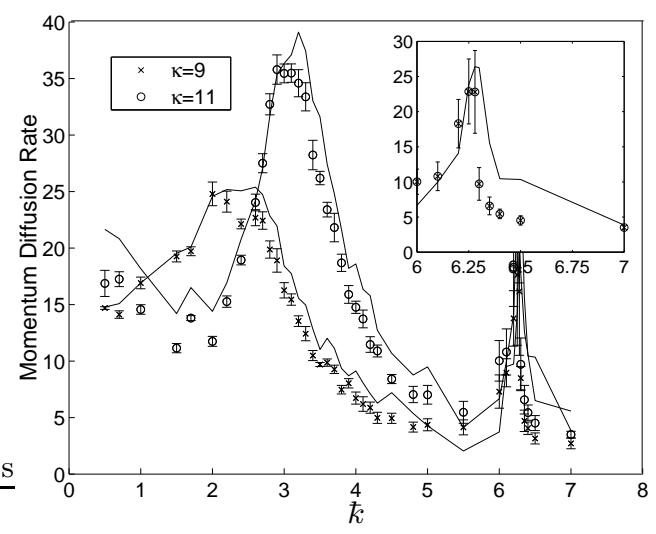

FIG. 4: Graph showing simulated late time momentum diffusion rates (points) and results from Eq.(6) (solid lines), with $\eta=10 \%$ and $\alpha=0.005$. The insert shows the peak near the quantum resonance at $k=2 \pi$ for $\kappa=11$.

large range of $k$ values by making assumptions about the form of $D_{0}(n)$. For example, we can assume that $D_{0}(0)=D_{0}(1)=\kappa^{2} / 4$, and that for $n \geq 2$ the diffusion rate starts at the initial quantum diffusion rate, $D_{q}$, and decays to zero exponentially with a time constant which depends on the quantum break time [6]. The main problem is in determining a form that works well near the quantum resonance, where the assumption of exponential relaxation in $D_{0}(n)$ breaks down and oscillations occur in the diffusion rate, as shown in Fig. 5 .

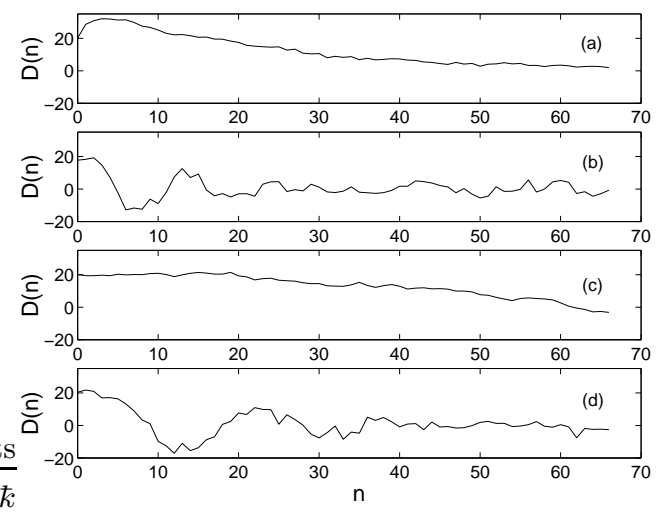

FIG. 5: Graph showing diffusion rates as a function of kick number for (a) $k=2$, (b) $k=6$, (c) $k=6.28$ and (d) $k=6.4$, with $\kappa=9$ and $\alpha=0.005$. Notice the inital quasilinear behaviour followed by exponential settling for lower $k$ values which contrasts with the oscillatory behaviour for $k$ values near the quantum resonance peak.

The behaviour which we observe in the late time rates across the quantum to classical transition for the atom optics kicked rotor is similar to that observed by Bhattacharya et al.14] for the DKR with a continuous posi- tion measurement, and leads to similar questions about the nature of the quantum to classical transition. The observation of such results from a real decoherence process is very interesting, and our simulations suggest that these results should be readily observable in laboratory experiments. In fact, hints of unusual behaviour in the momentum diffusion rates as a function of $k$ have already been observed in experiments with cold atoms [17, 18, 19].

We thank Kurt Jacobs for interesting and stimulating discussions and Andrew Doherty for providing the computer source code of Ref. [8], which formed the basis for our simulations. This work was supported by a grant (UOA016) from the Marsden Fund of the Royal Society of New Zealand.

Note added: After the completion of this work we learned of a cold atom KR experiment [M. B. d'Arcy et al., Phys. Rev. Lett. 87, 074102 (2001)] demonstrating enhanced diffusion resonances of the sort considered in this paper.

[1] G. Casati, B. V. Chirikov, F. M. Izrailev, and J. Ford, in Stochastic Behaviour in Classical and Quantum Hamiltonian Systems, Vol 93 of Lecture Notes in Physics, edited by G. Casati and J. Ford, Springer Berlin (1979).

[2] B. V. Chirikov, F. M. Izrailev, and D. L. Shepelyansky, Sov. Sci. Rev. Sec. C 2, 209 (1981).

[3] M. G. Raizen, Adv. At. Mol. Opt. Phys. 41, 43 (1999).

[4] E. Ott, T. M. Antonsen Jr., and J. D. Hanson, Phys. Rev. Lett. 53, 2187 (1984).

[5] T. Dittrich and R. Graham, Ann. Phys. 200, 363 (1990).

[6] D. Cohen, Phys. Rev. A. 44, 2292 (1991).

[7] S. Dyrting, Phys. Rev. A 53, 2522 (1996).

[8] A. C. Doherty et al., J. Opt. B: Quantum Semiclass. Opt. 2, 695 (2000).

[9] H. Ammann, R. Gray, I. Shvarchuck, and N. Christensen, Phys. Rev. Lett. 80, 4111 (1998).

[10] B. G. Klappauf, W. H. Oskay, D. A. Steck, and M. G. Raizen, Phys. Rev. Lett. 81, 1203 (1998).

[11] V. Milner, D. A. Steck, W. H. Oskay, and M. G. Raizen, Phys. Rev. E 61, 7223 (2000).

[12] D. A. Steck, V. Milner, W. H. Oskay, and M. G. Raizen, Phys. Rev. E. 62, 3461 (2000).

[13] K. Vant, G. Ball, and N. Christensen, Phys. Rev. E 61, 5994 (2000).

[14] T. Bhattacharya, S. Habib, K. Jacobs, and K. Shizume, quant-ph/0105086 (2001).

[15] W. H. Oskay et al., Opt. Comm. 179, 137 (2000).

[16] D. L. Shepelyansky, Physica 28D, 103 (1987).

[17] B. G. Klappauf, W. H. Oskay, D. A. Steck, and M. G. Raizen, Phys. Rev. Lett. 81, 4044 (1998).

[18] D. A. Steck, private communication.

[19] M. Williams, Master's thesis, University of Auckland (2001). 